\title{
Headache Diagnosis in Enhance patient satisfaction
}

\section{Agatha Christie}

${ }^{*}$ Corresponding Author: Agatha Christie, Department of Pharmacology, Nagasaki, Japan. Email: chagatha_44@gmail.net

Received date: January 18,2018 ;Accepted date : Febrauary 28,2018; Published date: August $19,2018$.

Citation for this Article: Agatha Christie, Headache Diagnosis in Enhance patient satisfaction . J Psychology and Mental Health

Care. Doi: 10.31579/2637-8892/037

Copyright : @ 2018 Agatha Christie. This is an open-access article distributed under the terms of The Creative Commons Attribution License, which permits unrestricted use, distribution, and reproduction in any medium, provided the original author and source are credited.

\section{Abstract \\ Introduction}

Doctors in primary care are responsible for diagnosing and managing patients with headache, but frequently lack confidence in doing so. We aimed to compare Family Practitioners' (FPs) diagnosis of headaches to classification based on a symptom questionnaire, and to describe how classification links to other important clinical features.

\section{Methods}

This was an observational study of patients attending primary care doctors for headache.

\section{Main outcome measures}

Patients completed a questionnaire including the Headache Impact Test, the Migraine Disability Assessment Score, the Hospital Anxiety and Depression Scale, the Illness Perceptions Questionnaire, a satisfaction scale, a service use inventory and a symptom questionnaire rated by two Practitioners with Special Interest (PSIs) in Headache.

\section{Results}

255 patients completed questionnaires. There was low agreement between FP diagnosis and classification using the symptom questionnaire. FPs frequently did not use the diagnosis migraine, when patient reported symptoms which justified this. FPs did not classify patients with $\geq 15$ days of headache separately as chronic daily headache $(\mathrm{CDH})$, and this could be because the classification system used does not have that code. Patients classified as $\mathrm{CDH}$ using the symptom questionnaire reported more disability, more symptoms of anxiety and depression (HADS), more service use, and less satisfaction with FP care.

\section{Conclusion}

Patients, who present with headache in primary care, tend to receive non-specific diagnoses. Having a system that would allow separate classification of people with headache of $\geq 15$ days a month might help FPs to explore and address associated features with patients in terms of disability, psychological co-morbidity and cost, and improve satisfaction with care.

\section{Keywords}

Diagnosis; Primary care; Headache; Migraine; Chronic daily headache

\section{Introduction}

Headache is common and $4 \%$ of adults consult their family practitioner (FP) for headache each year, with $97 \%$ managed entirely in primary care [1]. Doctors frequently express lack of confidence in diagnosing neurological conditions, which may partially be due to lack of appropriate clinical teaching [2]. We described the characteristics of patients with headache consulting FPs, and found nearly $30 \%$ had case-levels of anxiety [3]. Reasons FPs gave for referring to neurologists included the patient's anxiety about brain tumor, and the FP's lack of confidence in diagnosis [4]. We previously estimated UK service costs for people consulting with headache are $£ 956$ million and the total costs including lost production are $£ 4.8$ billion [5].

Guidelines on headache classification are disputed and have changed over time [6,7]. It is not clear how classification systems designed by neurologists and academic researchers can contribute to clinicians working in primary care. Comparison of FP diagnosis with expert classification has suggested under-diagnosis of migraine and underuse of migraine-specific management [8, 9]. Common headache types seen by FPs are migraine with and without aura, episodic tension-type headache $(\mathrm{TTH})$, and chronic daily headache $(\mathrm{CDH}=$ headache lasting on average for $\geq 4$ hours on $\geq 15$ days per month).

Secondary (sinister) headaches and cluster headache are rare. A major strength of UK primary care has been the computerization of patient records using the Read-code system, which has more than 30 codes for the common headache and migraine diagnoses. However, Read-codes were not designed to mirror or adapt to emerging criteria produced by specialists and researchers, such as the International Headache Society (IHS) classification of headache and migraine.

We aimed to describe the diagnoses made by FPs using Read-code data (http://www.connectingforhealth.nhs.uk /systemsandservices /data /uktc/readcodes), and compare them to a classification applied on the basis of symptoms reported by patients in a questionnaire, which was rated independently by two Practitioners with Special Interest (PSI) in headache. We aimed also to describe the extent to which PSIs' classifications were associated with other characteristics of headache consulters which may be important in management, including headache impact, disability, psychological state, service and lost-productivity costs and satisfaction with care.

\section{Patients and Methods}




\section{Practices and Patients}

Eighteen family practices, with 150 family practitioners (FPs) participated in the study. Practices were located in the South East region of the UK. The number of patients in the registered population aged 18-75 years was approximately 141,000. Eligible patients were those consulting for headache as a main or important problem. Study methods have been described in full elsewhere [3].

\section{Study Design}

To recruit patients, a designated person in each family practice identified each patient as they consulted for headache, classified according to pre-defined Read-codes. All 23 Read-codes including the word 'headache' were included in the search as well as all 11 including the word 'migraine', except for $115 \mathrm{E}=$ 'no history of migraine' unless it was accompanied by another headache Read-code. Patients identified as having consulted for headaches from the Readcode used by the FP during the consultation were invited to re-attend their practice to complete a questionnaire.

\section{Criteria-Based Classification}

Criteria were developed for this study based upon the IHS I (1998) guidelines [6]; with additional items from the Silberstein-Lipton criteria [10]. All patients completed a symptom questionnaire at their assessment, and patients could report up to three different headaches. Two PSIs independently rated questionnaire responses, allocating patients to classification groups depending on their headache symptoms, and assigning an overall classification where more than one headache was reported. The PSIs were blind to the Read-code recorded by the patient's FP. Using this method, patients were classified as having migraine with and without aura, headache on $\geq 15$ days a month classified as chronic daily headache $(\mathrm{CDH})$ with and without analgesic dependence, and 'other' headaches. The two PSI met to discuss their decisions when ratings differed. A migraine classification required two or more out of four of the following symptoms:- one sided, throbbing, worse with exertion, moderate of severe, plus one or more of nausea, photophobia, and phonophobia.

\section{Measures of Patients' Characteristics}

Patients' clinical characteristics were assessed using a self-report booklet of validated questionnaires including the Headache Impact Test (HIT-6), a valid and reliable six-item questionnaire designed to assess the impact headaches have on ability to function in the previous month [11], and the Migraine Disability Assessment Score (MIDAS), a valid and reliable five-item questionnaire requiring responses to five questions about disability associated with headache in the previous 3 months [12]. Anxiety and depression were measured using the Hospital Anxiety and Depression Scale (HADS), a valid and reliable 14-item self-report scale which has been widely used in community samples [13]. Patients were asked to report which of a list of 26 symptoms they experienced and considered to be connected with their headaches, which is part of the Illness Perceptions Questionnaire (IPQ-R) [14]. Patient satisfaction with treatment provided by their family practice was measured using one item from a reliable questionnaire developed for use in primary care [15]. Patients were asked to agree if they were satisfied and the 5-point Likert scale was anchored at $1=$ strongly disagree and $5=$ strongly agree. This scale was then dichotomized to disagree (1-3) and agree (4-5). Research associates collected information on the FPs' diagnosis and consultation frequency in the previous 3 months.

The services included were: contacts with FPs, neurologists, other medical specialists, contacts with other professionals (including complementary healthcare), scans undertaken (MRIs and CTs), and prescribed medication. For scans and FP contacts we asked about the number of times these had been received for headache and how many for other reasons. Unit costs were attached to the information on service use using nationally applicable figures [16]..The economic cost of lost work time was calculated by multiplying the lost days by the earnings that patients in the sample received (calculated as a daily figure).

Auctores Publishing - Volume1-10028 www.auctoresonline.org Page - 02
Not all patients stated their earnings and in these cases we obtained average figures for their job type and gender from official data [17].

\section{Data Analysis}

Data were analysed using SPSS version 8. Data were analysed using nonparametric tests for categorical variables, and with t-tests for continuous data where differences were compared between diagnostic groups. Statistical methods are described in details elsewhere [18].

\section{Results}

FPs' diagnosis from Read-codes of the 34 Read-codes available, nine were used by the FPs in this study, and we combined these codes, so that only the stem diagnosis was used. Of 255 patients, FPs classified 80 $(31 \%)$ patients as having migraine (FP-migraine) and $144(57 \%)$ as headache (FP-other headache), $23(9 \%)$ tension headache, and for the remaining $8(3 \%)$, headache was not classifiable.

\section{PSIs' criteria-based classification}

Using the criteria-based classification, 163/255 patients (63.9\%) reported experiencing a single type of headache, and 92 patients $(36.1 \%)$ experienced two or three different types of headaches. Ten patients $(4 \%)$ did not provide enough information for a classification. Where there was more than one type of headache reported, the higher category in terms of severity was applied. Using this strategy 152 (60\%) patients were classified as migraine, $78(31 \%)$ were classified as $\mathrm{CDH}$, and the remaining $15(6 \%)$ were classified in less common diagnostic groups, and subsequently excluded from further analysis.

\section{FP Read-code diagnosis compared to PSI criteria-based classification}

Table 1 shows that for migraine, there was agreement between the FPs' diagnosis and the PSI criteria-based classification in 55 cases ( $37 \%$ of 147 classified as migraine by PSIs). A further 92 (63\%) cases which were classified as migraine using the criteria, were diagnosed as other types of headache by FPs ( 84 other headache and eight tension headache). FPs did not have a code with which to identify people with headaches on $\geq 15$ days per month (CDH), and classified 45/76 (59\%) as headache, and 17/76 $(22 \%)$ patients as having migraine.

\begin{tabular}{|c|c|c|c|c|c|}
\hline \multirow{2}{*}{ 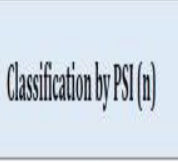 } & \multirow[b]{2}{*}{ Rriggiate } & \multicolumn{4}{|c|}{ 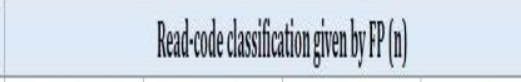 } \\
\hline & & 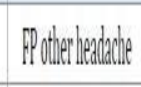 & Praximateralater & Vinarialyle & $\mathbb{N O H}$ \\
\hline ligigine & $\pi$ & A & 8 & $j$ & 12. \\
\hline OHH & 11 & 45 & H & ? & 18 \\
\hline 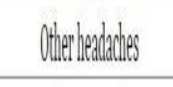 & 3 & II & 1 & 1 & 15 \\
\hline Indisitint & j & j & 0 & 1 & IN \\
\hline MOH & D & HH & $B$ & 8 & 37 \\
\hline
\end{tabular}

Table 1: Comparison between FPs' diagnosis and criteria-based classification

\section{Association of criteria-based classification with other patient characteristics}

Table 2 compares the criteria-based classification of patients with migraine and $\mathrm{CDH}$ ( $\geq 15$ days month). Compared to migraine, the $\mathrm{CDH}$ group had significantly more headache-related disability, significantly higher scores for anxiety and depression, and were more likely to be dissatisfied with the treatment received from their FP. Compared to migraine, the group with $\mathrm{CDH}$ had significantly higher service costs (migraine $£ 115$ (sd £156), CDH £164 (sd £194), bootstrapped 95\% CI of difference $£ 4$ to $£ 97$ ). 
Compared to migraine, the group with $\mathrm{CDH}$ had significantly higher total costs (migraine £475 (sd £1007), CDH £797 (sd £1438), bootstrapped $95 \%$ CI of difference $£ 6$ to $£ 680$ ).

\begin{tabular}{|c|c|c|c|c|}
\hline & \multicolumn{4}{|c|}{ Specialist diagnosis } \\
\hline & \multirow{2}{*}{$\begin{array}{c}\mathrm{CDH} \\
\mathrm{n}=78 \\
32.3(34.3)\end{array}$} & \multirow{2}{*}{$\begin{array}{c}\text { Migraine } \\
\mathbb{n}=152 \\
18.5(26.4)\end{array}$} & \multicolumn{2}{|c|}{ Mean difference (CI) \& P.valut } \\
\hline Headache disability(MIDAS) & & & $13.7\left(5.6 t_{0} 21.9\right)$ & 0.001 \\
\hline Number of headaches in previous 3 months & $55.0(29.0)$ & $16.0(18.1)$ & $38.9(32.5$ to 45.4$)$ & $<0.001$ \\
\hline Severity of pain $(1 \cdot 10)$ & $6.3(2.2)$ & $7.0(2.3)$ & $\cdot 0.8(-1.4$ to $\cdot 0.2)$ & 0.015 \\
\hline Headache impat(HIT-6) & $621(7.0)$ & $61.6(7.4)$ & $0.5(-1.6$ to 2.6$)$ & 0.65 \\
\hline Anxiety (HADS) $(0.21)^{*}$ & $8.5(4.8)$ & $7.2(4.2)$ & $1.3(0.02$ to 2.5$)$ & 0.047 \\
\hline Depression (HADS) $(0.21)^{*}$ & $5.8(4.1)$ & $4.0(3.4)$ & $1.8(0.7$ to 2.8$)$ & 0.001 \\
\hline Number of headache-related symptoms & $7.7(4.9)$ & $7.0(4.9)$ & $0.7(-0.7$ to 2.1$)$ & 0.34 \\
\hline Number of frisits to FP in previous 3 months & $1.6(1.3)$ & $1.8(1.9)$ & $\cdot 0.2(-0.6$ to 0.3$)$ & 0.52 \\
\hline Satisfeed with treatment from FP & $41(54 \%)$ & $97(68 \%)$ & $.14 \%(-28$ to -11$)$ & 0.04 \\
\hline Number of casses of anxiety & $27(37 \%)$ & $34(23 \%)$ & $14 \%(0.5$ to 27$)$ & 0.04 \\
\hline Number of cases of depression & $9(12 \%)$ & $8(6 \%)$ & $6 \%(-2 t 014)$ & 0.09 \\
\hline
\end{tabular}

Table 2: Patients' characteristics using criteria-based classification

\section{Discussion}

\section{Summary of main findings}

Compared to PSI's, FPs appear to underdiagnose migraine. As there were no codes for Chronic Daily Headache (CDH), FPs could not classify headaches in the same way as the PSI. Comparison between patients with migraine and $\mathrm{CDH}$, showed that the group with $\mathrm{CDH}$, reported more disability, more symptoms of anxiety and depression, higher costs and lower satisfaction with care.

\section{Strengths and the limitations of this study}

The study has compared a diagnosis recorded by a FP (with the patient present but with the limitations of time and the coding scheme), with classification made by practitioners with extra training and experiences (PSIs) who had access to and used responses to a symptom questionnaire. PSI having access and scoring a symptom questionnaire is clearly a different process from the clinical consultation, which can take account of other features, including FP not having special training, having limited time, and working with a coding scheme which is not designed to be consistent with criteria produced by specialists for academic research. It is possible that FPs gave their patients the optimal treatment irrespective of the Read-code used, assessing this was not the purpose of the study. However, interviews with a sample of the FPs in this study found that lack of clinician confidence and patient pressure were factors which influenced FPs in deciding to refer patients to specialists [4].

\section{Relationship to other studies}

In another family practice study, Weindels et al found that compared to patients with less frequent headache episodes, a group with frequent headaches ( $\geq 15$ days per month) were significantly more likely to have somatic problems, like gastroenterological and musculoskeletal disorders [18], as well as more psychiatric disorders and medication over-use [19]. Both frequent headache and co-morbidity were associated with lower quality of life [19]. Compared to hospital specialists, FPs potentially have more information about patients' other conditions and their management as a whole. However current diagnostic classification using the Read coding system does not make necessarily alert FPs to linking headache diagnosis with this other clinical information. In this context it is possible that psychological co-morbidity is under-diagnosed or not connected. Prescription of pain medication for headache and co-morbid conditions may contribute to a vicious cycle, with headache and pain symptoms becoming frequent and chronic. Our evidence may increase FPs awareness of frequent headache, and its co-morbidity, and stimulate FPs to identify and manage the co-morbidities of these patients more precisely.

Auctores Publishing - Volume1-10028 www.auctoresonline.org Page - 03

\section{Implications for clinical practice and research}

Our results suggest that FPs underuse migraine as a diagnosis. Previous findings suggest that when patients describe a few episodes of headache, they may not include symptoms like unilateral, pulsating pain, or think they have tension headache, and their doctors may not diagnose migraine [12]. Symptom diaries and questionnaires produce a longitudinal picture. FPs does not have a Read-code for $\mathrm{CDH}$, and have more problems managing patients with $\mathrm{CDH}$, because of associated co-morbidity. When we previously analysed and reported the quantitative data, in which this qualitative study was nested, we found a third of patients presenting in family practice were classified as chronic [3]. However when one of us described referrals to a headache clinic, two-thirds had CDH [20]. It is possible that failure to identify and address the disability, psychological morbidity and cost which can be associated with $\mathrm{CDH}$ may lead to dissatisfaction among some patients, who then apply pressure for referral to specialists. The read codes need a revision to include $\mathrm{CDH}$ because not having this code available limits the FP physician's ability to properly classify patients. If FPs are able to distinguish low from high frequency headache, this may help them to identify and manage the associated disability, psychological co-morbidity and cost associated with headache. Addressing these issues in primary care may have health gains for patients, enhance patient satisfaction, reduce referral, and reduce costs for patients and society [5]. This remains to be evaluated.

\section{References}

1. Latinovic R, Gulliford M, Ridsdale L (2006) Headache and migraine in primary care: consultation, prescription and referral rates in a large population. J Neurol Neurosurg Psychiatry 77: 385-387.

2. Ridsdale L, Massey R, Clark L (2007) No more neurophobia: making neurology teaching accessible to medical students. Practical Neurology 7: 116-123.

3. Ridsdale L, Clark LV, Dowson AJ, Goldstein LH, Jenkins L, et al. (2007) How do patients referred to neurologists for headache differ from those managed in primary care? Br J Gen Pract 57: 388-395.

4. Morgan M, Jenkins L, Ridsdale L (2007) Patient pressure for referral for headache: a qualitative study of GPs' referral behaviour. Br J Gen Pract 57: 29-35.

5. McCrone P, Seed PT, Dowson AJ, Clark LV, Goldstein LH, et al. (2011) Service use and costs for people with headache: a UK primary care study. J Headache Pain 12: 617-623.

6. Headache Classification Committee of the International Headache $\mathrm{S}$ (1988) Classification and diagnostic criteria for headache disorders, cranial neuralgias and facial pain. Cephalalgia 8: 1-96.

7. Headache Classification Subcommittee of the International Headache S (2004) The International Classification of Headache Disorders: 2nd edition. Cephalalgia 24: 1-160.

8. Cevoli S, D'Amico D, Martalletti P, Valguarnera F, Del Bene E, et al. (2009) Underdiagnosis and undertreatment of migraine in Italy: a survey of patients attending for the first time 10 headache centres. Cephalalgia 29:1285-1293

9. Tepper SJ, Dalhof CG, Dowson A, Newman L, Mansbach H, et al. (2004) Prevalence and diagnosis of migraine in patients consulting their physician with a complaint of headache: data from the Landmark Study. Headache 44: 856-864.

10. Silberstein SD, Lipton RB, Solomon S, Mathew NT (1994) Classification of daily and near-daily headaches: proposed revisions to the IHS criteria. Headache 34: 1-7.

11. Kosinski M, Bayliss MS, Bjorner JB, Ware JE, Jr., Garber WH, et al. (2003) A six-item short-form survey for measuring headache impact: the HIT-6. Qual Life Res 12: 963-974.

12. Stewart WF, Lipton RB, Whyte J, Dowson A, Kolodner K, et al. (1999) An international study to assess reliability of the Migraine Disability Assessment (MIDAS) score. Neurology 53: 988-994.

13. Zigmond AS, Snaith RP (1983) The Hospital Anxiety and Depression Scale. Acta Psychiatr Scand 67: 361-370.

14. Moss-Morris R, Weinman J, Petrie KJ, Horne R, Cameron LD, et al. (2002) The revised illness perception questionnaire (IPQ-R). Psychology \& Health 17: 1-16.

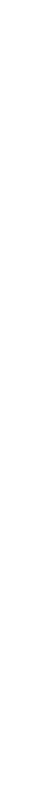


15. Baker R, Hearnshaw H (1993) Dialogue: a method for surveying patient satisfaction. In: Eli Lily National Audit Centre, Leicester, UK

16. Curtis L, Netten A (2004) Unit Costs of Health and Social Care. In: Personal Social Services Research Unit (PSSRU), Canterbury, PSSRU, University of Kent.

17. Office for National S (2004) Annual survey of hours and earnings (ASHE) - 2003 Results.

18. Wiendels NJ, Knuistingh NA, Rosendaal FR, Spinhoven P, Zitman FG, et al. (2006) Chronic frequent headache in the general population: prevalence and associated factors. Cephalalgia 26: 1434-1442.
19. Wiendels NJ, van Haestregt A, Knuistingh Neven A, Spinhoven P, Zitman FG, et al. (2006) Chronic frequent headache in the general population: comorbidity and quality of life Cephalalgia 26: 14431450.

20. Dowson AJ (2003) Analysis of the patients attending a specialist UK headache clinic over a 3-year period. Headache 43:14-18. 